\title{
Concurrent Use of Opioids with Other Central Nervous System-Active Medications Among Older Adults
}

\author{
Shirley Musich, PhD, ${ }^{1}$ Shaohung S. Wang, PhD, ${ }^{1}$ Luke B. Slindee, PharmD,2 \\ Joann Ruiz, RN, $\mathrm{MPH}^{3}$ and Charlotte S. Yeh, $\mathrm{MD}^{4}$
}

\begin{abstract}
The primary objective was to determine the prevalence and characteristics of older adults concurrently using opioids and other central nervous system (CNS)-active medications, and the specialties of providers who ordered the medications. A secondary objective was to document medication-related adverse effects associated with such concurrent drug use. Study populations were identified as older adults aged $\geq 65$ years with 1 year continuous medical and drug plan enrollment during 2017 and opioid use of $\geq 2$ prescriptions for $\geq 15$ days' supply. CNSactive medications included benzodiazepines, non-benzodiazepine hypnotics, muscle relaxants, antipsychotics, and gabapentinoids. Provider specialties were identified from the National Provider Identification database. Characteristics associated with opioids only, opioids plus 1 , and opioids plus $\geq 2$ additional CNS-active medications were determined using multinomial logistic regression. Outcome measures during 2017 included injurious falls/fractures and $\geq 3$ emergency room (ER) visits. Among eligible insureds $(\mathrm{N}=209,947), 57 \%$ used opioids only, $28 \%$ used opioids plus 1 additional CNS medication, and $15 \%$ used $\geq 2$ additional medications. About $60 \%$ of opioids and other concurrent CNS medications were prescribed by the same provider, generally a primary care provider. Benzodiazepines and gabapentinoids were most often used concurrently with opioids. Health status, insomnia, anxiety, depression, and low back pain had the strongest associations with concurrent medication use. Overall, concurrent use with $\geq 2$ CNS medications increased the likelihood of injurious falls/fractures or $\geq 3$ ER visits in this population by about $18 \%$ and $21 \%$, respectively. Both patients and providers may benefit from an awareness of adverse outcomes associated with concurrent opioid and other CNS-active medication use.
\end{abstract}

Keywords: older adults, opioids, CNS-active medications, injurious falls, emergency room use

\section{Introduction}

N 2016, The Centers for Disease Control and Prevention (CDC) issued guidelines for the use of opioids in the management of chronic pain. ${ }^{1}$ One of the 12 key recommendations focused on the concurrent prescribing of opioid pain medications along with other central nervous system (CNS)-active medications, especially benzodiazepines. ${ }^{1}$ The guidelines called for avoidance of concurrent prescribing of opioids and specifically benzodiazepines based on established adverse risks associated with concurrent use, notably respiratory depression, subsequently associated with overdose deaths, altered mental states affecting vehicle safety, and postural stability associated with falls/fractures in the those aged $\geq 65$ years. ${ }^{1}$ The CDC guidelines were supported by the American Geriatrics Society 2019 Updated Beers Criteria for Potentially Inappropriate Medications in Older Adults $^{2}$ and the US Federal Drug \& Food Administration Black Box warning of serious risks and death associated with combining opioid pain medications with benzodiazepines. ${ }^{3}$ Benzodiazepines and other CNS-active medications are often used in combination with opioids to augment analgesic effects and/or to manage insomnia, anxiety, and other mental health disorders frequently associated with chronic pain. ${ }^{4-12}$ Yet, despite the contraindications and warnings, concurrent use of opioids with other CNS-active medications, especially

\footnotetext{
${ }^{1}$ Research for Aging Populations, Optum, Ann Arbor, Michigan, USA.

${ }^{2}$ Informatics \& Data Science, Optum, Minneapolis, Minnesota, USA.

${ }^{3}$ Medicare \& Retirement, UnitedHealthcare, Minneapolis, Minnesota, USA.

${ }^{4}$ AARP Services, Inc., Washington, District of Columbia, USA.
}

(C) Shirley Musich et al. 2019; Published by Mary Ann Liebert, Inc. This Open Access article is distributed under the terms of the Creative Commons License (http://creativecommons.org/licenses/by/4.0), which permits unrestricted use, distribution, and reproduction in any medium, provided the original work is properly cited. 
benzodiazepines, remains common across age groups and within various patient settings. $8,9,13,14$

To date, concurrent use has no consistent definition in the scientific literature and has been variously defined as an overlap of 1 day, ${ }^{15-17} 30$ days, ${ }^{18}$ or 90 days ${ }^{7}$ when pharmaceutical claims were available or, more commonly, as during the same time period on surveys such as the National Ambulatory Medical Care Survey. ${ }^{8,9,13,14,19}$ Because the medications are widely utilized across all age groups, study samples are often population based including adults aged $\geq 18$ years with fewer studies focused on older adults aged $\geq 65$ years. $^{4,8,11,19-21}$ Furthermore, there is no consistent list of other CNS-active medications used with opioids. Concurrent use studies have been unilateral, such as those describing opioids with benzodiazepines ${ }^{7-10,14,17,18,22}$ or opioids with gabapentinoids, ${ }^{12,23,24}$ or have included various combinations of opioids with benzodiazepines, nonbenzodiazepine hypnotics, antipsychotics, muscle relaxants, and/or gabapentinoids. ${ }^{4-6,13,15,16,19}$ Consequently, the prevalence of concurrent use varies in the scientific literature depending on the age range of study samples, definitions of use, number of medications included, and the time frame of the study. Concurrent use has been reported from about $13 \%$ to $49 \%$ for opioids combined with a single CNSactive medication, ${ }^{6-8,10,13,16-18}$ such as benzodiazepines, non-benzodiazepine hypnotics, or antipsychotics, and from $25 \%$ to $55 \%$ for opioids combined with $>1$ CNS medication. $5,6,15,19$ Such concomitant pharmaceutical solutions are frequently offered as first-line treatment options whether patients present in a provider's office or in the emergency room (ER) for the treatment of chronic pain complicated with anxiety, insomnia, or other mental health issues. $5,6,8,11,14,18,19,22,25$

The providers prescribing concurrent opioids and other CNS-active medications have been characterized primarily as primary care providers (PCPs) who manage the regular care of their patients. $5,6,8,14,18,19$ However, the Veteran's Administration (VA), with a designed specialist referral system and computerized drug monitoring, has reported opioids to be more often prescribed by PCPs, with benzodiazepines and other mental health medications more often prescribed by mental health providers. ${ }^{5,15,25}$ As a result, more than $60 \%$ of concurrent use prescriptions were ordered by different providers. ${ }^{15}$ Thus, there is a debate as to whether care fragmentation across multiple providers is a major source of the concurrent use patterns or, in contrast, it is a lack of awareness and appreciation of the risks associated with concurrent use when prescribed by the same providers. ${ }^{7,15}$ When queried in qualitative interviews, physicians stated an awareness of the risks associated with concurrent use but cited a lack of alternatives in managing anxiety, insomnia, and pain. ${ }^{26,27}$ They referenced pharmaceutical management as a cost-efficient approach in preference to referrals to other specialists or hospitalizations, but voiced the need for more non-pharmacological alternatives. ${ }^{27}$

Attention to medication-related adverse effects associated with concurrent use of opioids and other CNS-activity medications has focused, as a priority, on respiratory depression and subsequent overdose deaths. 1,7,9,17,22 However, in one population-based study, of those who suffered overdose deaths from combined opioids and benzodiazepines, only $2 \%$ of those deaths were aged $\geq 65$ years. $^{22}$ The age group with the most deaths was $45-54$ years; 55-64 years had the largest percentage increase in deaths. ${ }^{22}$ Consistent with those results, disabled Medicare-eligible patients aged $<65$ years were more likely to be concurrent users than those $\geq 65$ years (36\% vs. $20 \%$ ). ${ }^{16}$ Thus, although overdose deaths associated with opioids and other CNS medications remain a relevant risk to older adults, altered mental states, postural instability, and possible dizziness resulting in increased falls and fractures may be most concerning. ${ }^{10,21}$ Risk for falls/fractures has been associated with opioids per se (hazard ratio 4.9 fractures upon initiation) ${ }^{21}$ and for combinations of opioids and benzodiazepines (self-reported falls; odds ratio [OR] 3.3). ${ }^{10}$ Subsequent to injury-related ER visits (OR 3.8), increased ER utilization also has been documented among older adults with chronic pain (OR 5.5). ${ }^{20}$ Unmanaged anxiety and panic attacks, sometimes presenting as chest pain, also are common reasons for increased and repeated ER utilization among older adults. ${ }^{28}$

Although recent research studies have considered the prevalence and characteristics associated with concurrent use of opioids with selected CNS-active medications, few have considered an inclusive list of CNS-active medications, used a measured definition of concurrent use or focused on the subpopulation of older adults aged $\geq 65$ year with Medicare Supplement plans (ie, Medigap). ${ }^{29}$ In the United States, government-funded Medicare covers adults aged 65 years and older, as well as those younger than age 65 and disabled. Medicare fee-for-service plans (about $70 \%$ of all Medicare plans) pay about $80 \%$ of medical expenditures for these individuals but offer minimal prescription drug benefits. Those enrolled in these Medicare plans are personally responsible for obtaining additional insurance plans to cover the remaining $20 \%$ of medical expenses (ie, Medicare Supplement or Medigap plans) and more inclusive prescription drug coverage (Medicare Part D plans). Although most (about 90\%) of those with original fee-for-service Medicare coverage have some type of supplemental insurance coverage, about $28 \%$ (currently about 10.2 million adults) have purchased Medigap coverage. ${ }^{29}$ Because this population may differ in demographic, socioeconomic, or health status characteristics from general older adult and/or specifically overall Medicare populations, it was of interest to examine the prevalence and characteristics associated with the concurrent use of opioids with other CNS-active medications in this study sample and to identify the numbers and specialties of providers ordering the prescriptions for these combinations of drugs. Inherent to a consideration of possible interventions that might enhance compliance with CDC guidelines is an understanding of whether prescriptions come from the same providers (ie, awareness of guidelines) or from different providers (ie, care coordination problem) including the identification of the specialty types (eg, primary care physician, psychiatrist, pain medicine) writing the prescriptions.

Thus, the primary objective was to estimate the prevalence and characteristics associated with concurrent use of opioids with other CNS-active medications in the study sample, along with the identification of the numbers and specialties of providers who were the sources of the various prescriptions. The secondary objective was to consider medication-related adverse outcomes associated with concurrent drug use, especially injurious falls/fractures and 
utilization of multiple ER visits. This research was reviewed and approved by the New England IRB \#120160532.

\section{Methods}

\section{Study sample}

In 2016, approximately 5 million Medicare insureds were covered by an AARP ${ }^{\mathrm{TM}}$ Medicare Supplement plan insured by UnitedHealthcare Insurance Company (UnitedHealthcare Insurance Company of New York for New York certificate holders). These plans are offered in all 50 states, Washington, DC, and various US territories. A sample of AARP Medicare Supplement insureds with AARP ${ }^{\mathrm{TM}}$ MedicareRx plans insured through UnitedHealthcare (about 55\% of insureds) with at least 2 opioid prescriptions and a cumulative day's supply $\geq 15$ days during 2017 was utilized to identify concurrent users of opioids with other CNS-active medications. Additional inclusion criteria for the study sample included: (1) 12-month continuous medical and drug plan enrollment during 2017, (2) provider specialty identification, (3) at least 65 years of age, and (4) exclusion of cancer or hospice patients. The final study sample that met the inclusion criteria included 209,947 insureds.

\section{Opioid users}

Opioids prescribed during 2017 were identified from National Drug Codes (NDCs) as recommended by the 2018 Health Effectiveness Data and Information Set (HEDIS) quality measures associated with opioid use. ${ }^{30}$ Opioid users were defined as those patients with at least 2 prescriptions for opioids and a cumulative days' supply of $\geq 15$ days. Opioids also were descriptively categorized into 6 mutually exclusive categories partially based on US Drug Enforcement Administration opioid drug schedules for acceptability of medical use, and potential for abuse or dependency. Those categories are: (1) long-acting; (2) short-acting, other Schedule II; (3) short-acting, oxycodone; (4) short-acting, hydrocodone; (5) short-acting, Schedule III-IV and nalbuphine; and (6) tramadol. ${ }^{31}$ Days of supply were calculated from prescriptions recorded in the pharmaceutical drug database during calendar year 2017.

\section{Other CNS-active medications}

Other CNS-active medications often prescribed concurrently with opioids in the management of pain, despite contraindications, included benzodiazepines, non-benzodiazepine hypnotics, muscle relaxants, antipsychotics, and gabapentinoids. These drug classes were defined from NDCs.

\section{Concurrent use definition}

Concurrent use of opioids with other CNS-active medications was defined as an overlap by prescription dates and days of supply for at least 30 days for both opioids and at least one of the other CNS medications (benzodiazepines, non-benzodiazepine hypnotics, muscle relaxants, antipsychotics, or gabapentinoids). Summary categories were subsequently defined as: opioid use only (at least 2 opioid prescriptions with a cumulative days' supply $\geq 15$ days); concurrent use of opioids plus 1 additional CNS medication; and concurrent use of opioids plus $\geq 2$ CNS medications. Additional subcategories of the CNS medications were not considered.

\section{Numbers and specialties of providers}

Numbers of providers and specialty types were identified from the National Provider Identifier (NPI) database maintained by the Centers for Medicare \& Medicaid Services (CMS). ${ }^{32}$ NPI data are part of a larger repository of provider information within the National Plan \& Provider Enumeration System database maintained by CMS. NPI is a unique identification number for covered health care providers. Covered health care providers and all health plans and health care clearinghouses must use the NPI in the administrative and financial transactions adopted under the Health Insurance Portability and Accountability Act. In addition, concurrent prescriptions of opioids with other CNS medications were identified as ordered by the same provider (eg, opioid and benzodiazepine prescriber) or by different providers (eg, opioid prescriber and benzodiazepine prescriber).

\section{Covariates}

Covariates were included to characterize categories of concurrent users of opioids and other CNS-active medications, and to adjust for other risk factors. These covariates included measures of demographics, socioeconomic factors, health status, and other characteristics taken from health plan eligibility and administrative medical claims.

Demographic questions included age and sex. Age groups were defined as: 65-69; 70-74; 75-79; 80-84; and $\geq 85$ years. Geographical location (Northeast, South, Midwest, or West); low (less than $15 \%$ nonwhite), medium (15\% to $59 \%$ nonwhite), and high ( $\geq 60 \%$ nonwhite) minority areas; and low $(<\$ 40,179)$, medium $(\$ 40,179$ to $<\$ 57,199)$, and high $(\geq \$ 57,199)$ median household income levels were geocoded from zip codes. AARP Medicare Supplement plan types were grouped by cost-sharing levels, including high-level coverage plans with minimal co-payments or deductibles, and all other plans. A measure of health services access was calculated as PCPs per 100,000 capita. Level of medical services utilization from medical claims was calculated as the Hierarchical Condition Category (HCC) score. ${ }^{33}$ The HCC score is used by CMS to risk adjust medical payments across various medical plans according to the health status of the different insured populations. HCC subgroups were defined as follows and utilized to control for health status: HCC scores <0.5; HCC scores 0.5 to $<1.2$; HCC scores 1.2 to $<2.8$; and HCC scores $\geq 2.8$.

\section{Prevalence of medical conditions}

Three chronic conditions related to concurrent use were defined from Charlson comorbidity index (CCI) diagnoses codes: chronic obstructive pulmonary disease, dementia, and rheumatoid arthritis. CCI is a measure of the risk of 1-year all-cause mortality attributable to selected comorbidities that also has been shown to be highly predictive of morbidity and health care expenditures. ${ }^{34}$

Three mental health conditions related to concurrent use were defined from Psychiatric Diagnosis Groups diagnoses 
codes: opioid use disorders (a measure of opioid dependency), major depression, and anxiety disorders. ${ }^{35}$

Musculoskeletal back pain was defined from back pain diagnoses codes after excluding all back pain associated with cancer, trauma, and drug abuse as defined by the HEDIS code specifications. ${ }^{30}$ Back pain diagnoses were documented at any time during the 12-month study period.

Additional possible medication-related adverse effects also were identified from diagnosis codes: pneumonia, respiratory distress, and serotonin syndrome. These potential adverse effects commonly highlighted in the opioid literature were included as associated with concurrent use with these medications, with the expectation that the conditions may be underestimated.

\section{Injurious falls/hip fractures}

Injurious falls requiring medical services or hip fractures, as a combined measure, were defined from suggested HEDIS diagnoses codes. ${ }^{30}$ Falls or hip fractures were documented from these selected diagnoses codes at any time during the 12-month study period. The risk for falls and subsequent fractures has been identified as independently associated with opioids and other CNS medications. The opioid only category was used as the reference, thus any association of increased fall/fractures would be associated with concurrent use with additional CNS medications.

\section{ER utilization}

The researchers did not have cause of ER visits, thus could only estimate the utilization of ER visits over the course of the study period. Based on the distribution and trends across concurrent use categories, multiple ER users were defined as $\geq 3$ visits during 2017 compared with occasional users with $0-2$ visits.

\section{Statistical models}

Demographic variables were unilaterally tested across the 3 concurrent use categories using chi-square or $t$ tests for categorical or continuous variables, respectively. Characteristics associated with opioids plus 1 and opioids plus $\geq 2$ additional CNS medications compared to opioid only users were determined using multinomial logistic regression models. Depression and anxiety were highly correlated; consequently, separate characteristics and outcomes regression models were developed. Covariates included all of those variables listed in Table 1. Variables with high correlations (eg, >0.5) were dropped from regression models. All analyses were completed using SAS Enterprise Guide Version 7.1 (SAS Institute Inc., Cary, NC, USA).

\section{Sensitivity analyses}

As a sensitivity analysis, the researchers also considered a 2-year continuous enrollment study sample 2016-2017 $(\mathrm{N}=195,388)$ using 2016 as a baseline year to identify new and continuing users of opioids and/or CNS medications during 2017. New users could not have any prescriptions for the drugs under study during calendar year 2016. If there were prescriptions prior to 2017, patients were considered as continuing users. Identifying numbers and specialties of providers for new and continuing users were used to confirm any differences in the patterns and provider types for concurrent use prescriptions.

\section{Results}

Overall, $31 \%$ of AARP Medicare Supplement insureds filled at least 1 opioid prescription in 2017 and, among these, $50 \%$ used at least 2 prescriptions of opioids for $\geq 15$ days $(\mathrm{N}=350,936)$. From this initial study sample, 9\% $(\mathrm{N}=31,433)$ were excluded because of continuous enrollment criteria; $27 \%(\mathrm{~N}=95,436)$ were excluded because of cancer diagnoses or hospice care; $3 \%(\mathrm{~N}=8968)$ were excluded for age $<65$ years or missing sex; and $1 \%(\mathrm{~N}=5152)$ were excluded because of missing provider specialty data. After these exclusions, the final study population included 209,947 insureds (30\% of opioid users) in the following concurrent use categories: $57 \%$ opioids only; $28 \%$ opioids plus 1 additional CNS medication; $15 \%$ opioids plus $\geq 2$ additional CNS medications (Table 1). In sensitivity analyses, $29 \%(\mathrm{~N}=56,415)$ of the 2-year sample were considered new users of opioids (at least 2 prescriptions for $\geq 15$ days) and/or other CNS medications. Of these, $14 \%$ used opioids plus 1 and $5 \%$ used opioids plus $\geq 2$ additional CNS medications in their first year of drug exposure. Because the results for new and continuing users were similar to the 1-year sample, the researchers opted to show only the results using the 2017 one-year study sample with relevant comparisons noted in the text.

As a group, those with at least 2 opioid prescriptions lasting $\geq 15$ days were mostly female, 70-74 years of age, white, high income, lived in the South region, and were in high-coverage medical plans (Table 1). The most common opioid prescriptions were for short-acting hydrocodone, tramadol, or short-acting oxycodone. Overall, CNS medications used most often in combination with opioids were gabapentinoids, benzodiazepines, and non-benzodiazepine hypnotics. Likewise, for opioids plus 1 or opioids plus $\geq 2$ additional CNS medications, gabapentinoids and benzodiazepines were the most commonly used prescriptions in combination with opioids.

\section{Numbers and specialties of providers}

Generally, about $60 \%$ (range $43 \%$ to $68 \%$ ) of concurrent opioids and CNS medications were prescribed by the same provider, most often a PCP (ie, family medicine, internal medicine, nurse practitioner) (Table 2). For the remaining $40 \%$ with opioids and CNS medication prescriptions ordered by different providers, the pattern was similar with the exception of pain medicine for opioids and psychiatry for CNS medications (Table 3). Nevertheless, the majority of prescriptions for both opioids and CNS medications, despite being sourced from different providers, were ordered by PCPs.

In sensitivity analyses, numbers and specialty types of providers were similar for both new and continuing users, reflecting the conclusions of the 1-year study sample. New users of concurrent drugs had a majority of prescriptions ordered by the same providers (range $41 \%$ to $65 \%$ ), most often a PCP. Thus, concurrent prescriptions appear to be prescribed by PCPs managing the various physical and mental health symptoms of their patients, without referrals to other specialties. 
Table 1. Unadjusted Demographic Characteristics for Concurrent Use of Opioids and Other Central Nervous System-Active Medications: Opioids Only, Opioids Plus One, and Opioids Plus $\geq$ Two

\begin{tabular}{|c|c|c|c|c|}
\hline & $\begin{array}{l}\text { All Mean } \\
\quad \text { or } \%\end{array}$ & $\begin{array}{l}\text { Opioids only } \\
\text { Mean or } \%\end{array}$ & $\begin{array}{l}\text { Opioids plus } 1 \\
\text { Mean or } \%\end{array}$ & $\begin{array}{c}\text { Opioids plus } \geq 2 \\
\text { Mean or } \%\end{array}$ \\
\hline Number & 209,947 & 119,744 & 58,463 & 31,740 \\
\hline \multicolumn{5}{|l|}{ Sex } \\
\hline Male & 31.0 & 33.5 & 28.9 & 25.3 \\
\hline Female & 69.0 & 66.5 & 71.1 & 74.7 \\
\hline Age (years) & 75.6 & 75.9 & 75.8 & 74.2 \\
\hline $65-69$ & 25.7 & 24.6 & 24.9 & 31.6 \\
\hline $70-74$ & 26.8 & 26.3 & 26.3 & 29.3 \\
\hline $75-79$ & 19.6 & 19.8 & 19.9 & 18.2 \\
\hline $80-85$ & 12.4 & 12.7 & 13.1 & 10.3 \\
\hline$\geq 85$ & 15.5 & 16.6 & 15.9 & 10.6 \\
\hline \multicolumn{5}{|l|}{ Minority (from zip codes) } \\
\hline Low & 48.1 & 48.6 & 48.0 & 46.8 \\
\hline Medium & 46.9 & 46.3 & 47.3 & 48.7 \\
\hline High & 3.4 & 3.6 & 3.3 & 3.1 \\
\hline \multicolumn{5}{|l|}{ Median Income (from zip codes) } \\
\hline Low & 18.0 & 17.2 & 18.9 & 19.1 \\
\hline Medium & 38.1 & 37.6 & 38.9 & 38.7 \\
\hline High & 43.6 & 44.8 & 41.9 & 42.0 \\
\hline \multicolumn{5}{|l|}{ Region } \\
\hline Midwest & 17.5 & 18.1 & 17.4 & 15.7 \\
\hline Northeast & 16.3 & 17.3 & 15.6 & 13.9 \\
\hline South & 43.6 & 41.9 & 44.5 & 48.1 \\
\hline West & 22.3 & 22.4 & 22.3 & 22.1 \\
\hline \multicolumn{5}{|l|}{ Access to health care } \\
\hline PCP per 100,000 capita & 131.0 & 131.7 & 130.6 & 129.3 \\
\hline \multicolumn{5}{|l|}{ Plan type } \\
\hline High & 78.0 & 77.4 & 78.1 & 80.1 \\
\hline Medium & 2.9 & 2.9 & 3.1 & 2.7 \\
\hline Other & 19.1 & 19.7 & 18.9 & 17.2 \\
\hline \multicolumn{5}{|l|}{ HCC Score } \\
\hline$\leq 0.50$ & 19.4 & 21.9 & 16.6 & 15.2 \\
\hline 0.50 to $<1.20$ & 40.1 & 41.9 & 38.6 & 36.0 \\
\hline 1.20 to $<2.80$ & 31.9 & 29.4 & 34.4 & 36.9 \\
\hline$\geq 2.8$ & 8.6 & 6.8 & 10.4 & 12.0 \\
\hline \multicolumn{5}{|l|}{ CCI Conditions } \\
\hline Dementia & 10.2 & 8.8 & 11.0 & 14.2 \\
\hline Chronic obstructive pulmonary disease & 30.2 & 27.7 & 32.1 & 36.3 \\
\hline Rheumatoid arthritis & 8.8 & 8.0 & 9.6 & 10.6 \\
\hline \multicolumn{5}{|l|}{ PDG Conditions } \\
\hline Opioid use disorders & 9.2 & 6.9 & 10.5 & 15.2 \\
\hline Major depression & 21.3 & 16.3 & 23.2 & 36.6 \\
\hline Anxiety disorders & 22.0 & 14.9 & 26.0 & 41.7 \\
\hline Insomnia $\mathrm{dx}$ & 11.0 & 6.6 & 13.4 & 23.4 \\
\hline Pneumonia $\mathrm{dx}$ & 10.3 & 8.9 & 11.2 & 13.7 \\
\hline Respiratory distress $\mathrm{dx}$ & 28.9 & 27.1 & 30.2 & 33.3 \\
\hline Serotonin syndrome $\mathrm{dx}$ & 0.5 & 0.4 & 0.6 & 1.0 \\
\hline Low back pain $\mathrm{dx}$ & 46.4 & 41.5 & 51.0 & 56.3 \\
\hline Injury fall/hip fracture $\mathrm{dx}$ & 11.8 & 11.6 & 11.3 & 13.4 \\
\hline Hip fracture $\mathrm{dx}$ & 2.4 & 2.5 & 2.3 & 2.3 \\
\hline Injurious fall dx & 10.8 & 10.6 & 10.4 & 12.4 \\
\hline Any inpatient hospitalization & 32.3 & 33.2 & 29.8 & 33.4 \\
\hline \multicolumn{5}{|l|}{ Number of ER visits } \\
\hline 0 & 53.5 & 54.0 & 54.8 & 49.1 \\
\hline $1-2$ & 33.8 & 34.2 & 32.6 & 34.1 \\
\hline$\geq 3$ & 12.8 & 11.8 & 12.6 & 16.7 \\
\hline
\end{tabular}


TABle 1. (CONTINUED)

\begin{tabular}{lcccc}
\hline & $\begin{array}{c}\text { All Mean } \\
\text { or \% }\end{array}$ & $\begin{array}{c}\text { Opioids only } \\
\text { Mean or \% }\end{array}$ & $\begin{array}{c}\text { Opioids plus } 1 \\
\text { Mean or \% }\end{array}$ & $\begin{array}{c}\text { Opioids plus } \geq 2 \\
\text { Mean or \% }\end{array}$ \\
\hline Opioid category & & & & \\
$\quad$ Long acting & 9.6 & 5.4 & 12.6 & 20.1 \\
Short acting, other Schedule II & 5.1 & 3.9 & 5.5 & 8.8 \\
Short acting, oxycodone & 31.7 & 30.8 & 30.1 & 38.0 \\
Short acting, hydrocodone & 49.2 & 48.8 & 48.7 & 8.6 \\
Short acting, Schedule III-IV & 9.7 & 10.9 & 8.3 & 37.1 \\
$\quad$ Tramadol & 46.5 & 49.9 & 44.6 & \\
Concurrent use of opioids with other & & & & 66.0 \\
CNS medications & 20.8 & 0.0 & 38.8 & 63.1 \\
Opioids and gabapentinoids & 18.4 & 0.0 & 31.9 & 49.3 \\
Opioids and benzodiazepines & 12.3 & 0.0 & 17.5 & 35.7 \\
Opioids and non-benzo hypnotics & 7.8 & 0.0 & 8.7 & 14.5 \\
Opioids and muscle relaxants & 3.1 & 0.0 & 3.1 & \\
Opioids and antipsychotics & & & \\
\hline
\end{tabular}

Notes: All variables are statistically significant at $P<0.0001$. Missing categories deleted for brevity.

CCI, Charlson comorbidity index; CNS, central nervous system; dx, diagnoses codes; ER, emergency room; HCC, Hierarchical Condition Category; PCP, primary care provider; PDG, Psychiatric Diagnosis Groups.

Table 2. Concurrent Opioids and Other Central Nervous System-Active Medications Provider Type Prescribers: SAme Provider

\begin{tabular}{lc}
\hline & Same \\
Provider type prescriber & provider $\%$ \\
\hline Opioids \& Benzodiazepines $(\mathbf{N}=\mathbf{2 3 , 2 0 3})$ & $\mathbf{6 0}$ \\
Family Medicine & 38 \\
Internal Medicine & 38 \\
Nurse Practitioner & 6 \\
All others & 19 \\
Opioids \& Gabapentinoids $(\mathbf{N}=\mathbf{2 7 , 0 1 3})$ & $\mathbf{6 2}$ \\
Family Medicine & 30 \\
Internal Medicine & 28 \\
Pain Medicine & 11 \\
Nurse Practitioner & 8 \\
Physician Assistant & 5 \\
All others & 19 \\
Opioids \& Non-Benzodiazepine Hypnotics & $\mathbf{5 5}$ \\
(N=14,303) & \\
Family Medicine & 38 \\
Internal Medicine & 37 \\
Nurse Practitioner & 6 \\
All others & 19 \\
Opioids \& Muscle Relaxants $(\mathbf{N}=\mathbf{1 1 , 1 1 9})$ & $\mathbf{6 8}$ \\
Family Medicine & 26 \\
Internal Medicine & 20 \\
Pain Medicine & 16 \\
Nurse Practitioner & 9 \\
Physician Assistant & 8 \\
All others & 20 \\
Opioids \& Antipsychotics $(\mathbf{N}=\mathbf{2 7 6 2})$ & $\mathbf{4 3}$ \\
Family Medicine & 38 \\
Internal Medicine & 35 \\
Nurse Practitioner & 8 \\
Geriatric Medicine & 5 \\
All others & \\
\hline Notes All o & \\
\hline
\end{tabular}

Notes: All others includes summed total of all other provider types individually less than $5 \%$ of prescriptions.
Characteristics associated with opioids plus 1 and opioids plus $\geq 2$ CNS medications

The characteristics with the strongest associations with concurrent use of opioids with CNS medications were poor health status (high HCC scores); mental health conditions of insomnia, anxiety and depression; and youngest age group 65-69 years (Table 4). Other significant characteristics of note associated with both levels of concurrent drug use included female, low minority (white), low income, diagnosed low back pain, and more likely to experience an injurious fall/fracture (opioids plus $\geq 2$ only).

\section{Adjusted injurious falls/fractures and multiple ER utilization}

Opioids plus 1 additional CNS medication had mini$\mathrm{mal} / \mathrm{no}$ association with increased injurious falls/fractures or multiple ( $\geq 3$ visits) ER utilization. However, concurrent use of opioids plus $\geq 2$ additional CNS medications, adjusted for the variables in Table 1, maintained a significant association with both increased injurious falls/fractures and increased multiple ER utilization: by $18 \%$ and $21 \%$, respectively (depression models), and by $18 \%$ and $14 \%$, respectively (anxiety models) (Tables 5 and 6).

In sensitivity analyses, injurious falls/fractures and multiple ER utilization for continuing users were increased significantly for opioids plus $\geq 2$ additional CNS medications by $33 \%$ and $33 \%$, respectively, and by $45 \%$ and $39 \%$, respectively, for new users. Opioids plus 1 CNS medication, although statistically significant in the 2-year models, was minimally associated with either of the adverse outcomes (1\% to $7 \%$ increased).

\section{Discussion}

In this study sample of AARP Medicare Supplement insureds with extended opioid use, 57\% used opioids only, $28 \%$ used opioids plus 1 additional CNS medication, and $15 \%$ used $\geq 2$ additional medications. Although definitions 
Table 3. Concurrent Opioids and Other Central Nervous System-Active Medications Provider Type Prescribers: Different Providers

\begin{tabular}{|c|c|c|c|}
\hline Provider type prescribers & $\begin{array}{c}\text { Different } \\
\text { provider } \%\end{array}$ & Provider type prescribers & $\begin{array}{c}\text { Different } \\
\text { provider } \%\end{array}$ \\
\hline Opioids $(N=15,447)$ & 40 & Benzodiazepines & 40 \\
\hline Pain Medicine & 22 & Internal Medicine & 29 \\
\hline Family Medicine & 12 & Family Medicine & 27 \\
\hline Internal Medicine & 12 & Psychiatry & 18 \\
\hline Nurse Practitioner & 10 & Nurse Practitioner & 7 \\
\hline Physician Assistant & 9 & All others & 19 \\
\hline Orthopedic Surgery & 7 & & \\
\hline Physical Medicine \& Rehab & 6 & & \\
\hline Rheumatology & 6 & & \\
\hline All others & 17 & & \\
\hline Opioids $(\mathrm{N}=16,631)$ & 38 & Gabapentinoids & 38 \\
\hline Pain Medicine & 18 & Internal Medicine & 22 \\
\hline Family Medicine & 16 & Family Medicine & 21 \\
\hline Internal Medicine & 16 & Nurse Practitioner & 12 \\
\hline Nurse Practitioner & 11 & Neurology & 11 \\
\hline Physician Assistant & 9 & Physician Assistant & 7 \\
\hline Orthopedic Surgery & 6 & Pain Medicine & 5 \\
\hline All others & 25 & All others & 22 \\
\hline Opioids $(N=11,566)$ & 45 & Non-Benzodiazepine Hypnotics & 45 \\
\hline Pain Medicine & 22 & Internal Medicine & 31 \\
\hline Family Medicine & 11 & Family Medicine & 29 \\
\hline Internal Medicine & 11 & Psychiatry & 14 \\
\hline Nurse Practitioner & 10 & Nurse Practitioner & 8 \\
\hline Physician Assistant & 9 & All others & 18 \\
\hline Orthopedic Surgery & 8 & & \\
\hline Physical Medicine \& Rehab & 6 & & \\
\hline Rheumatology & 6 & & \\
\hline All others & 16 & & \\
\hline Opioids $(N=5304)$ & 32 & Muscle Relaxants & 32 \\
\hline Pain Medicine & 22 & Family Medicine & 21 \\
\hline Family Medicine & 17 & Internal Medicine & 18 \\
\hline Internal Medicine & 14 & Nurse Practitioner & 17 \\
\hline Nurse Practitioner & 11 & Physician Assistant & 10 \\
\hline Physician Assistant & 8 & Neurology & 9 \\
\hline Orthopedic Surgery & 5 & Pain Medicine & 6 \\
\hline \multirow[t]{2}{*}{ All others } & 24 & Rheumatology & 5 \\
\hline & & All others & 14 \\
\hline Opioids $(N=3668)$ & 57 & Antipsychotics & 57 \\
\hline Internal Medicine & 18 & Psychiatry & 45 \\
\hline Pain Medicine & 17 & Internal Medicine & 16 \\
\hline Family Medicine & 16 & Family Medicine & 14 \\
\hline Nurse Practitioner & 14 & Nurse Practitioner & 7 \\
\hline Physician Assistant & 7 & Neurology & 6 \\
\hline All others & 28 & All others & 12 \\
\hline
\end{tabular}

Notes: All others includes summed total of all other provider types individually less than $5 \%$ of prescriptions.

of "concurrent use" for opioids and other CNS-active medications varied and fewer studies focused exclusively on study populations aged $\geq 65$ years, the prevalence of those in this study with documented concurrent use of $\geq 30$ days was in general agreement with previous publications (ie, about $20 \%$ concurrent use for a single medication; about $40 \%$ for 22). ${ }^{5-8,10,13,15-19}$ As in other studies, those who used opioids and other CNS medications concurrently often were taking other medications to manage mental health problems of anxiety, insomnia, or depression associated with their chronic pain management. ${ }^{4-11}$ Not surprisingly, the most commonly used concurrent medications with opioids were gabapentinoids and benzodiazepines. ${ }^{6,12,15,19,25}$ Use of gabapentinoids has increased dramatically in recent years to augment the analgesic effect of opioids, perhaps associated with pressure to use lower dosages of opioids. ${ }^{12,24}$

About $60 \%$ of opioids and other CNS-active medications were prescribed by the same providers, most often a PCP. Thus, although care fragmentation with multiple prescriptions ordered across multiple providers may contribute to the problem, ${ }^{7,15}$ these data would suggest that PCPs managing the regular care of their patients are the source of much concurrent prescribing despite guidelines and warnings to the contrary. It would appear that whether patients 
Table 4. Characteristics Associated with Opioids Plus One and Opioids Plus $\geq$ Two Additional Central Nervous System-Active Medications Compared to Opioids Only

\begin{tabular}{|c|c|c|c|c|c|c|c|c|}
\hline \multirow[b]{3}{*}{ Variable } & \multicolumn{4}{|c|}{ Depression models } & \multicolumn{4}{|c|}{ Anxiety models } \\
\hline & \multicolumn{2}{|c|}{ Opioids plus 1} & \multicolumn{2}{|c|}{ Opioids plus $\geq 2$} & \multicolumn{2}{|c|}{ Opioids plus 1} & \multicolumn{2}{|c|}{ Opioids plus $\geq 2$} \\
\hline & Odds ratio & $\mathrm{P}$ value & Odds ratio & $\mathrm{P}$ value & Odds ratio & $\mathrm{P}$ value & Odds ratio & $\mathrm{P}$ value \\
\hline Female & 1.31 & $<0.0001$ & 1.63 & $<0.0001$ & 1.27 & $<0.0001$ & 1.56 & $<0.0001$ \\
\hline Age $65-69$ & 1.26 & $<0.0001$ & 2.67 & $<0.0001$ & 1.26 & $<0.0001$ & 2.69 & $<0.0001$ \\
\hline Age $70-74$ & 1.17 & $<0.0001$ & 2.07 & $<0.0001$ & 1.16 & $<0.0001$ & 2.08 & $<0.0001$ \\
\hline Age $75-79$ & 1.11 & $<0.0001$ & 1.59 & $<0.0001$ & 1.11 & $<0.0001$ & 1.59 & $<0.0001$ \\
\hline Age 80-84 & 1.07 & 0.0003 & 1.26 & $<0.0001$ & 1.07 & 0.0009 & 1.25 & $<0.0001$ \\
\hline Minority low & 1.14 & $<0.0001$ & 1.18 & $<0.0001$ & 1.13 & $<0.0001$ & 1.15 & $<0.0001$ \\
\hline Minority medium & 1.16 & $<0.0001$ & 1.22 & $<0.0001$ & 1.14 & $<0.0001$ & 1.20 & $<0.0001$ \\
\hline Income low & 1.16 & $<0.0001$ & 1.13 & $<0.0001$ & 1.15 & $<0.0001$ & 1.11 & $<0.0001$ \\
\hline Income middle & 1.09 & $<0.0001$ & 1.05 & 0.004 & 1.08 & $<0.0001$ & 1.04 & 0.01 \\
\hline Midwest & 0.93 & $<0.0001$ & 0.80 & $<0.0001$ & 0.93 & $<0.0001$ & 0.80 & $<0.0001$ \\
\hline Northeast & 0.90 & $<0.0001$ & 0.76 & $<0.0001$ & 0.89 & $<0.0001$ & 0.75 & $<0.0001$ \\
\hline West & 0.96 & 0.005 & 0.86 & $<0.0001$ & 0.98 & 0.13 & 0.89 & $<0.0001$ \\
\hline Plan type: medium & 1.07 & 0.04 & 0.95 & 0.22 & 1.07 & 0.03 & 0.96 & 0.35 \\
\hline Plan type: others & 0.99 & 0.65 & 0.95 & 0.007 & 1.00 & 0.75 & 0.96 & 0.01 \\
\hline PCP per 100,000 capita & 1.00 & 0.003 & 1.00 & $<0.0001$ & 1.00 & 0.005 & 1.00 & $<0.0001$ \\
\hline HCC Score 0.50 to $<1.20$ & 1.28 & $<0.0001$ & 1.51 & $<0.0001$ & 1.28 & $<0.0001$ & 1.55 & $<0.0001$ \\
\hline HCC Score 1.20 to $<2.80$ & 1.66 & $<0.0001$ & 2.34 & $<0.0001$ & 1.67 & $<0.0001$ & 2.44 & $<0.0001$ \\
\hline HCC Score $\geq 2.8$ & 2.21 & $<0.0001$ & 3.32 & $<0.0001$ & 2.23 & $<0.0001$ & 3.53 & $<0.0001$ \\
\hline Rheumatoid arthritis $\mathrm{dx}$ & 1.08 & $<0.0001$ & 1.09 & 0.0003 & 1.09 & $<0.0001$ & 1.10 & $<0.0001$ \\
\hline Opioid use disorders & 1.41 & $<0.0001$ & 1.79 & $<0.0001$ & 1.37 & $<0.0001$ & 1.71 & $<0.0001$ \\
\hline Depression $\mathrm{dx}$ & 1.37 & $<0.0001$ & 2.27 & $<0.0001$ & - & - & - & - \\
\hline Anxiety dx & - & - & - & - & 1.80 & $<0.0001$ & 3.21 & $<0.0001$ \\
\hline Insomnia $\mathrm{dx}$ & 2.06 & $<0.0001$ & 3.70 & $<0.0001$ & 1.97 & $<0.0001$ & 3.46 & $<0.0001$ \\
\hline Low back pain $\mathrm{dx}$ & 1.49 & $<0.0001$ & 1.86 & $<0.0001$ & 1.48 & $<0.0001$ & 1.83 & $<0.0001$ \\
\hline Injurious falls/fractures $\mathrm{dx}$ & 0.93 & $<0.0001$ & 1.12 & $<0.0001$ & 0.92 & $<0.0001$ & 1.11 & $<0.0001$ \\
\hline
\end{tabular}

Notes: Reference categories include: male; age $\geq 85$; minority high; income high; South; plan type: high; HCC Score <0.50; no rheumatoid arthritis; no opioid use disorders; no depression; no anxiety; no insomnia; no low back pain, and no injurious falls/fractures.

$\mathrm{dx}$, diagnosis code; HCC, Hierarchical Condition Category; PCP, primary care provider.

present in the PCP office or the ER, providers are asked to address pain and mental health-related symptoms for which there are few alternatives. ${ }^{5,6,9,14,18,19,22,25-27}$ Contrary to the VA system, with drug-monitoring systems and a referral system to other specialists, ${ }^{5,10,15,26}$ the general PCP has no such resources. Thus, pharmaceutical solutions remain firstline treatment options in the general care of broader populations of adults to manage pain along with anxiety, insomnia, depression and the multiple chronic conditions common among older adults. ${ }^{4,5,8,14,18,19,25}$

Characteristics associated with concurrent use of opioids and other CNS medications included anxiety, insomnia, depression, low back pain, and poorer health, but younger age groups 65-69 years. Other characteristics of note included female, white, lower income, and more likely to suffer an injurious fall/fracture. These characteristics are generally consistent across research studies. ${ }^{4-11,13,14,17}$ That concurrent use of medication increased with younger age groups is noteworthy as these individuals age into Medicareeligible coverage plans. Problems with anxiety, depression, and insomnia have been consistently associated with pain management but to date have few alternatives to pharmacological treatments. ${ }^{4-11,27}$

Medication-related adverse effects measured in this study included respiratory distress, pneumonia, serotonin syndrome, and injurious fall/fractures. Of these conditions, only injurious falls/fractures demonstrated a significant association with concurrent use of opioids plus $\geq 2$ CNS medications. The magnitude of increased injurious falls/fractures in the present study, about 20\%, was less than the parallel Yorborough et al study ${ }^{10}$ that reported more than 3 times higher falls among concurrent users. Their study on a younger study sample (59 years) used self-reported falls as the outcome, which may explain the difference in part. In the present study sensitivity analysis, new users of these drugs had increased falls/fractures by about $50 \%$. This increased likelihood is consistent with suggestions that the risk of falls/fractures is comparatively highest upon initiation and dissipates somewhat over time. ${ }^{21}$ The researchers did not know the reasons associated with ER utilization, and hence could not track drug-drug interactions or specific anxiety-related visits. But, if one assumes that CNS-active medication treatments are consistent with the documented diagnoses of anxiety, insomnia, and depression, the $20 \%$ increased multiple ER visits would be consistent with other studies, and with the suggestion that many avoidable ER visits are associated with anxiety and pain-related symptomology. $10,14,17,20,25,27$

At the provider level, suggestions have been made for improved education and policy changes to guide providers in avoiding concurrent use of these medications. $7,14,23,27$ Better integration of pharmacists with access to prescription 
Table 5. Adjusted Odds Ratios for Injurious Falls/Fractures Associated with Opioids and Other Central Nervous System-Active Medications: Plus One and Plus $\geq$ Two

\begin{tabular}{|c|c|c|c|c|}
\hline \multirow[b]{2}{*}{ Variable } & \multicolumn{2}{|c|}{ Depression model Injurious falls/fractures } & \multicolumn{2}{|c|}{ Anxiety model Injurious falls/fractures } \\
\hline & Odds ratio & $\mathrm{P}$ value & Odds ratio & $\mathrm{P}$ value \\
\hline Opioids plus 1 CNS medication & 0.96 & 0.02 & 0.95 & 0.003 \\
\hline Opioids plus $\geq 2$ CNS medications & 1.18 & $<0.0001$ & 1.18 & $<0.0001$ \\
\hline Female & 1.30 & $<0.0001$ & 1.31 & $<0.0001$ \\
\hline Age 70-74 & 1.35 & $<0.0001$ & 1.34 & $<0.0001$ \\
\hline Age $75-79$ & 1.80 & $<0.0001$ & 1.79 & $<0.0001$ \\
\hline Age $80-84$ & 2.62 & $<0.0001$ & 2.60 & $<0.0001$ \\
\hline Age $\geq 85$ & 3.45 & $<0.0001$ & 3.43 & $<0.0001$ \\
\hline Minority low & 1.09 & 0.02 & 1.09 & 0.02 \\
\hline Minority medium & 1.07 & 0.06 & 1.07 & 0.05 \\
\hline Income low & 1.02 & 0.35 & 1.00 & 0.97 \\
\hline Income middle & 0.99 & 0.40 & 0.98 & 0.21 \\
\hline Midwest & 1.12 & $<0.0001$ & 1.14 & $<0.0001$ \\
\hline Northeast & 1.17 & $<0.0001$ & 1.17 & $<0.0001$ \\
\hline West & 0.99 & 0.69 & 1.01 & 0.61 \\
\hline Plan type: medium & 0.83 & $<0.0001$ & 0.83 & $<0.0001$ \\
\hline Plan type: others & 0.93 & 0.0002 & 0.93 & $<0.0001$ \\
\hline PCP per 100,000 capita & 1.00 & $<0.0001$ & 1.00 & $<0.0001$ \\
\hline Rheumatoid arthritis $\mathrm{dx}$ & 1.16 & $<0.0001$ & 1.19 & $<0.0001$ \\
\hline Opioid use disorders & 1.54 & $<0.0001$ & 1.56 & $<0.0001$ \\
\hline Depression $\mathrm{dx}$ & 2.09 & $<0.0001$ & - & - \\
\hline Anxiety dx & - & - & 1.74 & $<0.0001$ \\
\hline Insomnia $\mathrm{dx}$ & 1.31 & $<0.0001$ & 1.33 & $<0.0001$ \\
\hline Low back pain $\mathrm{dx}$ & 0.36 & $<0.0001$ & 0.35 & $<0.0001$ \\
\hline
\end{tabular}

Notes: Reference categories included: male; age 65-69; minority high; income high; South; plan type: high; no rheumatoid arthritis; no opioid use disorders; no depression; no anxiety; no insomnia; and no low back pain.

CNS, central nervous system; dx, diagnosis code; PCP, primary care provider.

monitoring systems could help alert providers of drug overlaps and interactions, especially from different providers. More available referral patterns could provide PCPs access to pain medicine and mental health specialists as options for patient care. ${ }^{5,6,14,18,19,25}$ Perhaps, most importantly, there are few non-pharmacological alternatives available for the treatment of anxiety, insomnia, and chronic pain issues. Non-pharmacological approaches have shown success among older adults and include: cognitivebehavioral therapies (CBT) for anxiety, insomnia, and/or pain; mindfulness; acupuncture; and physical therapy options. ${ }^{14,18,19,36-38}$ In the PCP environment, however, multisession interventions have lacked practicality because of time and resource limitations. In addition, many published interventions remain within research settings, involve small study samples, and consequently have not been widely implemented.

Direct-to-patient communications also have been suggested to inform patients of the risks associated with these medication combinations relative to the severity of their symptoms. ${ }^{19,26}$ Once patients have concurrent prescriptions, safe tapering of opioids and/or CNS mediations requires patient-provider agreement, a stepwise, time-consuming protocol, and, although demonstrating some success, has been characterized by a high recidivism rate. ${ }^{39}$ In the VA environment, depression management among noncancer pain patients has been associated with opioid cessation. ${ }^{40}$ Finally, health plans, including Medicare and/or Medicare Advantage, may need to consider more inclusive coverage to include more mental health benefits, more non- pharmacological options such as CBT, and better access to pain and mental health specialists. ${ }^{9}$

\section{Limitations}

This study has some limitations. The study population of AARP Medicare Supplement insureds may not generalize to all older adults or other Medicare, Medicare Advantage or Medicare Supplement beneficiaries. Pharmacy databases confirmed prescription purchases but there was no indication of whether patients actually consumed the drugs as directed. Mental health issues were identified from diagnosis codes and/or medication use, thus depression, insomnia and anxiety were likely underreported. Medication-related adverse effects were identified from diagnosis codes and may be underestimated. Strengths of the study included a large study population that applied a measured definition of 30 days of overlap for concurrent use of a more inclusive list of CNS medications. Provider specialties were documented from the external NPI database. Characteristics of concurrent medication users and selected medication-related adverse effects of falls/fractures and multiple ER use provided comprehensive information to define the opioid and CNS medication concurrent use problem in consideration of possible solutions.

\section{Conclusions}

Overall, in this study sample of Medicare Supplement insureds with extended opioid use, about $40 \%$ used $\geq 1$ concurrent CNS-active medications. Anxiety, depression, 
Table 6. Adjusted Odds Ratios for Three or More Emergency Room Visits Associated with Opioids and Other Central Nervous System-Active Medications: Plus One and Plus $\geq$ Two

\begin{tabular}{|c|c|c|c|c|}
\hline \multirow[b]{2}{*}{ Variable } & \multicolumn{2}{|c|}{ Depression models $\geq 3$ ER visits } & \multicolumn{2}{|c|}{ Anxiety models $\geq 3$ ER visits } \\
\hline & Odds ratio & $\mathrm{P}$ value & Odds ratio & $\mathrm{P}$ value \\
\hline Opioid plus 1 CNS medication & 0.97 & 0.08 & 0.94 & $<0.0001$ \\
\hline Opioid plus $\geq 2 \mathrm{CNS}$ medications & 1.21 & $<0.0001$ & 1.14 & $<0.0001$ \\
\hline Female & 0.97 & 0.03 & 0.95 & 0.002 \\
\hline Age $70-74$ & 1.18 & $<0.0001$ & 1.18 & $<0.0001$ \\
\hline Age $75-79$ & 1.48 & $<0.0001$ & 1.48 & $<0.0001$ \\
\hline Age $80-84$ & 1.88 & $<0.0001$ & 1.88 & $<0.0001$ \\
\hline Age $\geq 85$ & 2.14 & $<0.0001$ & 2.15 & $<0.0001$ \\
\hline Minority low & 0.90 & 0.001 & 0.89 & 0.0003 \\
\hline Minority medium & 0.91 & 0.004 & 0.91 & 0.003 \\
\hline Income low & 1.05 & 0.02 & 1.03 & 0.20 \\
\hline Income middle & 1.04 & 0.009 & 1.03 & 0.03 \\
\hline Midwest & 1.04 & 0.03 & 1.06 & 0.004 \\
\hline Northeast & 0.99 & 0.64 & 0.99 & 0.56 \\
\hline West & 1.04 & 0.04 & 1.07 & 0.0002 \\
\hline Plan type: medium & 0.90 & 0.007 & 0.90 & 0.009 \\
\hline Plan type: others & 0.91 & $<0.0001$ & 0.91 & $<0.0001$ \\
\hline PCP per 100,000 capita & 1.00 & 0.003 & 1.00 & 0.01 \\
\hline Rheumatoid arthritis $\mathrm{dx}$ & 1.35 & $<0.0001$ & 1.38 & $<0.0001$ \\
\hline Opioid use disorders & 1.98 & $<0.0001$ & 1.96 & $<0.0001$ \\
\hline Depression $\mathrm{dx}$ & 2.24 & $<0.0001$ & - & - \\
\hline Anxiety $\mathrm{dx}$ & - & - & 2.30 & $<0.0001$ \\
\hline Insomnia $\mathrm{dx}$ & 1.40 & $<0.0001$ & 1.37 & $<0.0001$ \\
\hline Low back pain $\mathrm{dx}$ & 0.84 & $<0.0001$ & 0.83 & $<0.0001$ \\
\hline
\end{tabular}

Notes: Reference categories included: male; age 65-69; minority high; income high; South; plan type: high; no rheumatoid arthritis; no opioid use disorders; no depression; no anxiety; no insomnia; and no low back pain.

CNS, central nervous system; dx, diagnosis code; ER, emergency room; PCP, primary care provider.

and insomnia were prominent mental health conditions associated with such use. Contrary to expectations, prescriptions were most often from the same provider, generally a PCP. Increased falls/fractures and multiple ER use were associated with concurrent use of $\geq 2$ CNS medications. Better awareness of the adverse effects associated with concurrent opioid and other CNS medication use may benefit both patients and providers. More evidence-based nonpharmacological options to address anxiety, insomnia, and pain symptoms are warranted.

\section{Author Disclosure Statement}

Drs Musich, Wang, and Slindee, and Ms Ruiz are all employed by UnitedHealth Group and have stock with UnitedHealth Group. Dr Yeh is employed by AARP Services, Inc. However, their compensation was not dependent upon the results obtained in this research, and the investigators retained full independence in the conduct of this research.

\section{Funding Information}

This work was funded by the AARP Medicare Supplement Insurance Program.

\section{References}

1. Dowell D, Haegerich TM, Chou R. CDC guideline for prescribing opioids for chronic pain-United States, 2016. MMWR Recomm Rep 2016;65:1-49.

2. American Geriatrics Society Beers Criteria ${ }^{\circledR}$ Update Expert Panel. American Geriatrics Society 2019 updated AGS
Beers Criteria ${ }^{\circledR}$ for potentially inappropriate medication use in older adults. J Am Geriatr Soc 2019;67:674-694.

3. US Food \& Drug Administration. FDA Drug Safety Communication: FDA warns about serious risks and death when combining opioid pain or cough medicines with benzodiazepines. 2016. https://www.fda.gov/media/99761/ download Accessed May 13, 2019.

4. Cotton BP, Lohman MC, Brooks JM, et al. Prevalence of and factors related to prescription opioids, benzodiazepines, and hypnotics among Medicare home health recipients. Home Healthc Now 2017;35:304-313.

5. Barry DT, Sofuoglu M, Kerns RD, Wiechers IR, Rosenheck RA. Prevalence and correlates of co-prescribing psychotropic medications with long-term opioid use nationally in the Veterans Health Administration. Psychiatry Res 2015;227:324-332.

6. Deyo RA, Smith DH, Johnson ES, et al. Opioids for back pain patients: primary care prescribing patterns and use of services. J Am Board Fam Med 2011;24:717-727.

7. Hernandez I, He M, Brooks MM, Zhang Y. Exposureresponse association between concurrent opioid and benzodiazepine use and risk of opioid-related overdose in Medicare Part D beneficiaries. JAMA Netw Open 2018;1:e180919.

8. Rhee TG. Co-prescribing of benzodiazepines and opioids in older adults: rates, correlates, and national trends. J Gerontol A Biol Sci Med Sci 2018. DOI: 10.1093/gerona/gly283 [Epub ahead of print].

9. Ladapo JA, Larochelle MR, Chen A, et al. Physician prescribing of opioids to patients at increased risk of overdose from benzodiazepine use in the United States. JAMA Psychiatry 2018;75:623-630. 
10. Yarborough BJH, Stumbo SP, Stoneburner A, et al. Correlates of benzodiazepine use and adverse outcomes among patients with chronic pain prescribed long-term opioid therapy. Pain Med 2019;20:1148-1155.

11. Kim M, Mitchell SH, Gatewood M, et al. Older adults and high-risk medication administration in the emergency department. Drug Healthc Patient Saf 2017;9:105-112.

12. Peckham AM, Fairman KA, Sclar DA. All-cause and drugrelated medical events associated with overuse of gabapentin and/or opioid medications: a retrospective cohort analysis of a commercially insured US population. Drug Saf 2018;41:213-228.

13. Larochelle MR, Zhang F, Ross-Degnan D, Wharam JF. Trends in opioid prescribing and co-prescribing of sedative hypnotics for acute and chronic musculoskeletal pain: 20012010. Pharmacoepidemiol Drug Saf 2015;24:885-892.

14. Hirschtritt ME, Delucchi KL, Olfson M. Outpatient, combined use of opioid and benzodiazepine medications in the United States, 1993-2014. Prev Med Rep 2017;9:49-54.

15. Mosher HJ, Richardson KK, Lund BC. Sedative prescriptions are common at opioid initiation: an observational study in the Veterans Health Administration. Pain Med 2018;19:788-792.

16. Centers for Medicare \& Medicaid Services. Concurrent use of opioids and benzodiazepines in a Medicare Part D population. 2016. https://www.cms.gov/Medicare/PrescriptionDrug-Coverage/PrescriptionDrugCovContra/Downloads/ Concurrent-Use-of-Opioids-and-Benzodiazepines-in-aMedicare-Part-D-Population-CY-2015.pdf Accessed February $6,2019$.

17. Sun EC, Dixit A, Humphreys K, Darnall BD, Baker LC, Mackey S. Association between concurrent use of prescription opioids and benzodiazepines and overdose: retrospective analysis. BMJ 2017;356:j760.

18. Simon J, Gehret J, Stolzenberg D, et al. Concomitant use of opioids and benzodiazepines in the outpatient setting: a retrospective study. PM R 2018;pii: S1934-1482(18)30926-2.

19. Gerlach LB, Olfson M, Kales HC, Maust DT. Opioids and other central nervous system-active polypharmacy in older adults in the United States. J Am Geriatr Soc 2017;65:20522056.

20. Castillo EM, Brennan JJ, Howard J, et al. Factors associated with geriatric frequent users of emergency departments. Ann Emerg Med 2019;74:270-275.

21. Miller M, Stürmer T, Azrael D, Levin R, Solomon DH. Opioid analgesics and the risk of fractures in older adults with arthritis. J Am Geriatr Soc 2011;59:430-438.

22. Jones CM, McAninch JK. Emergency department visits and overdose deaths from combined use of opioids and benzodiazepines. Am J Prev Med 2015;49:493-501.

23. Peckham AM, Evoy KE, Covvey JR, Ochs L, Fairman KA, Sclar DA. Predictors of gabapentin overuse with or without concomitant opioids in a commercially insured U.S. population. Pharmacotherapy 2018;38:436-443.

24. Gomes T, Juurlink DN, Antoniou T, Mamdani MM, Paterson JM, van den Brink W. Gabapentin, opioids, and the risk of opioid-related death: a population-based nested case-control study. PLoS Med 2017;14:e1002396.

25. Dark T, Flynn HA, Rust G, Kinsell H, Harman JS. Epidemiology of emergency department visits for anxiety in the United States: 2009-2011. Psychiatr Serv 2017;68:238244.

26. Hawkins EJ, Malte CA, Hagedorn HJ, et al. Survey of primary care and mental health prescribers' perspectives on reducing opioid and benzodiazepine co-prescribing among veterans. Pain Med 2017;18:454-467.

27. Kim HS, McCarthy DM, Hoppe JA, Mark Courtney D, Lambert BL. Emergency department provider perspectives on benzodiazepine-opioid coprescribing: a qualitative study. Acad Emerg Med 2018;25:15-24.

28. Centers for Disease Control and Prevention. National Hospital Ambulatory Medical Care Survey: 2016 Emergency Department Summary Tables. https://www.cdc.gov/nchs/ data/nhamcs/web_tables/2016_ed_web_tables.pdf Accessed May 13, 2019.

29. AHIP Center for Policy and Research. Trends in Medigap Enrollment and Coverage Options, 2014. https://www.ahip .org/wp-content/uploads/2016/04/MedigapEnrollmentReport_ Linked.pdf Accessed May 1, 2017.

30. National Committee for Quality Assurance. HEDIS ${ }^{\circledR}$ Measures included in the 2018 Quality Rating System (QRS). www.ncqa.org/hedis-quality-measurement/hedis-measures/ hedis-2018 Accessed March 16, 2018.

31. United States Drug Enforcement Administration. Drug Schedules. https://www.dea.gov/druginfo/ds.shtml Accessed July 5, 2018.

32. Center for Medicare \& Medicaid Services. National Provider Identifier Standard: Data dissemination. https:// www.cms.gov/Regulations-and-Guidance/AdministrativeSimplification/NationalProvIdentStand/DataDissemination .html Accessed May 3, 2019.

33. Pope GC, Kautter J, Ingber MJ, Freeman S, Sekar R, Newhart C. Evaluation of the CMS-HCC Risk Adjustment Model, 2011. https://www.cms.gov/Medicare/Health-Plans/ MedicareAdvtgSpecRateStats/downloads/evaluation_risk_ adj_model_2011.pdf Accessed March 3, 2017.

34. Charlson ME, Pompei P, Ales KL, et al. A new method of classifying prognostic comorbidity in longitudinal studies: development and validation. J Chronic Dis 1987;40: 373-383.

35. Ashcraft ML, Fries BE, Nerenz DR, et al. A psychiatric patient classification system. An alternative to diagnosisrelated groups. Med Care 1989;27:543-557.

36. Hofmann SG, Smits JA. Cognitive-behavioral therapy for adult anxiety disorders: a meta-analysis of randomized placebocontrolled trials. J Clin Psychiatry 2008;69:621-632.

37. Perach R, Allen CK, Kapantai I, et al. The psychological wellbeing outcomes of nonpharmacological interventions for older persons with insomnia symptoms: a systematic review and meta-analysis. Sleep Med Rev 2019;43:1-13.

38. Lozier CC, Nugent SM, Smith NX, et al. Correlates of use and perceived effectiveness of non-pharmacologic strategies for chronic pain among patients prescribed long-term opioid therapy. J Gen Intern Med 2018;33(suppl 1):46-53.

39. Vanderlip ER, Sullivan MD, Edlund MJ, et al. National study of discontinuation of long-term opioid therapy among veterans. Pain 2014;155:2673-2679.

40. Scherrer JF, Salas J, Sullivan MD, et al. Impact of adherence to antidepressants on long-term prescription opioid use cessation. Br J Psychiatry 2018;212:103-111.

Address correspondence to: Shirley Musich, PhD Research for Aging Populations Optum 315 East Eisenhower Parkway, Suite 305 Ann Arbor, MI 48108 USA

E-mail: shirley.musich@optum.com 